\title{
Adenosine Dysfunction and Adenosine Kinase in Epileptogenesis
}

\author{
Detlev Boison*
}

\author{
RS Dow Neurobiology Laboratories, Legacy Research, Portland, OR 97232, USA
}

\begin{abstract}
Traditionally, epilepsy has been considered to be a disorder of neuronal dysfunction. Based on this dogma, drug development efforts have largely focused on neurocentric model systems to screen for compounds that affect the function of neurons. Unfortunately, about $30 \%$ of all patients with epilepsy - or more than 20 million worldwide - are refractory to classical neurocentric pharmacotherapy. The failure of neurocentric pharmacotherapy in epilepsy requires radical rethinking and the search for novel therapeutic targets. Research from recent years suggests that epilepsy is a disorder of astrocyte dysfunction. Astrocytes are key regulators of the brain's own anticonvulsant adenosine. Thus, any dysfunction in astrocyte metabolism will drastically affect the brain's ability to control excitability via adenosinergic neuromodulation. This review will focus on the astrocyte-based enzyme adenosine kinase (ADK) as the key regulator of synaptic adenosine. Astrogliosis - a pathological hallmark of the epileptic brain - leads to overexpression of the adenosineremoving enzyme ADK and therefore to adenosine deficiency. Evidence from transgenic animals demonstrates that overexpression of ADK per se is sufficient to trigger seizures. Consequently, pharmacological inhibition of ADK is very effective in suppressing seizures that are refractory to classical antiepileptic drugs. The recent definition of ADK as rational target to predict and to prevent seizures in epilepsy has prompted the development of focal adenosine augmentation therapies (AATs) that have been designed to selectively reconstitute adenosinergic signalling within an area of astrogliosisbased adenosine-dysfunction. This therapeutic challenge has experimentally been met with polymeric or stem cell based brain implants to afford the focal delivery of adenosine.
\end{abstract}

Keywords: Epilepsy, astrogliosis, epileptogenesis, adenosine, adenosine kinase, cell therapy, gene therapy.

\section{INTRODUCTION}

Most clinically used antiepileptic drugs (AEDs) have been designed to affect neuronal function by directly affecting neuronal ion channels, presynaptic release mechanisms, or neuronal receptor function $[1,2]$. Many of these therapeutic drug effects are based on solidly studied mechanisms. For example, the gabapentinoids gabapentin and pregabalin exert their anticonvulsant activity by binding to $\alpha 2 \delta$ subunits of voltage-gated calcium channels $[3,4]$, whereas mutations in those subunits lead to spontaneous seizures in mice [5, 6]. Despite solid characterization of AEDs and the addition of new compounds to the clinical armamentarium, it is estimated that more than $30 \%$ of all patients with epilepsy remain refractory to treatment with neurocentric therapeutic strategies or suffer from intolerable side effects [2]. Therefore, alternative treatment strategies have been explored (and shown to be efficient) that range from electrical stimulation $[7,8]$ to the ketogenic diet [9-12]. Recent findings suggest that these alternative treatments are at least in part based on mechanisms related to adenosine $[12,13]$. Moreover, astrocytes, the key regulators of synaptic adenosine, appear to be crucially involved in seizure regulation. This review will first discuss the role of astrocyte dysfunction in general and then focus on adenosine and its regulation within the context of epilepsy, whereas other components of purinergic signalling will be covered in different reviews of this issue.

*Address correspondence to this author at the Robert Stone Dow Neurobiology Laboratories, Legacy Research, $1225 \mathrm{NE} 2^{\text {nd }}$ Ave, Portland, OR, 97232, USA; Tel: (503) 413-1754; Fax: (503) 413-5465;

E-mail: dboison@downeurobiology.org

\section{AN ASTROCYTIC BASIS OF EPILEPSY}

Astrogliosis - the pathological proliferation and hypertrophy of astrocytes - is a pathological hallmark of the epileptic brain and alterations in distinct astrocyte membrane channels, receptors and transporters have all been associated with the epileptic state [14]. The concept of the tripartite synapse in which astrocytic processes ensheath neuronal synapses and contribute to the regulation of synaptic transmission [15] is an excellent explanation how astrocytes can influence and modify neuronal function and, consequently, dysfunctional gliotransmission plays an important role in the pathophysiology of epilepsy [16]. An astrocytic basis of epilepsy has first been proposed by Nedergaard and colleagues based on the demonstration that paroxysmal depolarization shifts that are involved in the initiation of seizures can be triggered by the release of glutamate from extrasynaptic sources or by photolysis of caged $\mathrm{Ca}^{2+}$ in astrocytes [17]. In these studies three AEDs - valproate, gabapentin, and phenytoin - decreased the ability of astrocytes to transmit $\mathrm{Ca}^{2+}$ signalling. It was further demonstrated that astrocytic glutamate was released via SNARE-dependent exocytosis of glutamate-containing vesicles [18]. Together, these studies suggested that astrocytic glutamate release may play an epileptogenic role in the initiation of epileptic seizures under pathological conditions. In line with these findings, gene expression changes in astrocytes in temporal lobe epilepsy were suggested to contribute to an increased release of glutamate by astrocytes [19]. Therefore, astrogliosis in epilepsy might be a likely contributing factor to the glutamate overflow that is a characteristic of the epileptic brain [20]. However, the notion that astrocyte-derived glutamate contributes 
to epileptiform activity has recently been challenged based on pharmacological studies performed in hippocampal slice models of epileptiform activity [21]. In these studies, TTX blocked ictal- and interictal-like epileptiform activity without affecting slow inward currents that depend on astrocytic glutamate release. In contrast, NMDA receptor antagonists blocked the astrocyte dependent slow inward currents, but did not prevent the generation of epileptiform events. Thus, based on this study, the non-synaptic glutamate release from astrocytes appears not to be necessary for the generation of epileptiform activity in vitro.

In addition to the direct contribution of astrocytes to calcium and glutamate signalling, astrocytes have the capability to affect several epileptogenic and ictogenic mechanisms more indirectly. Thus, specific inflammatory pathways are chronically activated during epileptogenesis and astrocytes have been shown to sustain those inflammatory processes by activating the interleukin 1 beta system [22]. Astrocytes of the epileptic brain are also characterized by structural reorganization and a loss of astrocytic domain organization, morphological alterations that are thought to contribute to the structural basis for recurrent excitation of the epileptic brain [23]. Astroglial gap junctions provide an activitydependent intercellular pathway for the delivery of energetic metabolites from blood vessels to distal neurons and thereby play critical roles in coupling neuronal function to the metabolic state of an organism [24]. In particular, astrocytes play important roles in ATP-signalling [16] and astrocytic connexin 43 hemichannels may contribute significantly to extrasynaptic ATP efflux [25]. It was recently hypothesized that ATP released from astrocytes in response to neuronal activity is a source of surround inhibition to adjacent neurons, a mechanism that could prevent seizure propagation [16]. The identification of non-neuronal and non-chemical synaptic signalling pathways described here offers new and promising targets for the therapy of epilepsy [26].

\section{ASTROCYTES AND ADENOSINE}

Astrocytes are key regulators of the brain's endogenous anticonvulsant adenosine and an astrocyte-based adenosine cycle has been proposed [27, 28]. Astrocytes are a major source of ATP that can be released into the synaptic cleft via hemichannels [25] or via regulated synaptic release [29]. Synaptic ATP is rapidly cleaved by a series of ectonucleotidases into adenosine $[30,31]$. In pioneering experiments by Newman [32] it was demonstrated in rat retina that activation of glial cells reduced the firing rate of neurons that displayed spontaneous spike activity. This effect could be blocked by an adenosine $A_{1} R$ antagonist or by inhibitors of ATP cleavage [32]; these findings demonstrated that activated glial cells can inhibit neurons in the retina by the release of ATP, which is converted to adenosine by ectoenzymes and subsequently activates neuronal adenosine receptors. In an elegant series of experiments Haydon and coworkers demonstrated that astrocyte-derived ATP regulates synaptic strength and plasticity [29]. In these experiments the authors used inducible transgenic mice that expressed a dominant-negative SNARE domain selectively in astrocytes to block the release of transmitters (including ATP) from these glial cells. It was demonstrated that astrocytes tonically suppressed synaptic transmission by releasing adenosine triphosphate as a precursor of adenosine, an effect that was abolished in the mutant mice. These results and related studies, which demonstrated regulated ATP release from astrocytes through lysosome exocytosis [33], indicate that astrocytes - via regulating the release of a precursor of adenosine (i.e. ATP) - play important roles in the regulation and coordination of synaptic strength, plasticity, and synaptic networks [29, 34]. In line with these findings, the inhibition of gliotransmission in dominant-negative SNARE transgenic mice attenuated the accumulation of sleep pressure, assessed by measuring the slow wave activity of the EEG during NREM sleep, and prevented cognitive deficits associated with sleep loss [35]. Findings from this study indicate that astrocytes modulate the accumulation of sleep pressure and its cognitive consequences through a pathway involving $A_{1}$ receptors [35]. Subsequent studies using adenosine microelectrode biosensors demonstrated directly in neurochemical online measurements that ATP is a precursor of synaptic adenosine [36, 37]. Using similar approaches, it was further demonstrated that astrocytic adenosine kinase (ADK) regulates basal synaptic adenosine levels and seizure activity but not activity-dependant adenosine release in the hippocampus [38].

Astrocytes contain two types of equilibrative nucleoside transporters [39], which facilitate the rapid equilibration of synaptic and intra-astrocytic levels of adenosine [27, 28]. In contrast to neurotransmitters that all have their respective energy-driven transporter-mediated re-uptake system to terminate synaptic activity of the neurotransmitter, an equivalent system lacks for adenosine. Instead, the intracellular astrocyte-based [40] enzyme ADK appears to fulfill the role of a metabolic re-uptake system for adenosine. Therefore, astrocytic ADK (see subsequent chapters for details) plays a key role for the regulation of synaptic levels of adenosine [41]. The critical contribution of astrocytes for the regulation of synaptic adenosine was further corroborated in neuron / astrocyte coculture systems, in which NMDA-evoked neuronal adenosine release was subject to metabolism by added astrocytes [42].

\section{ADENOSINE KINASE AND ADENOSINE}

Adenosine levels can be reduced by adenosine deaminase (ADA, forming inosine), which shows highest expression levels in tongue, cells lining the intestinal tract, and thymus, whereas its expression in brain is rather low and limited to specific nuclei [43-45]. Adenosine kinase (ADK, E.C. 2.7.1.20) is responsible for the enzymatic phosphorylation of adenosine into AMP [46] (Fig. 1). In a comparative study performed in brain slices it was demonstrated that inhibition of ADK, but not of ADA, led to increases in ambient adenosine resulting in presynaptic inhibition [47]. It was realized more than 30 years ago that mammalian cells deficient in ADK are capable of excreting accumulating purines including adenosine [48]. ADK belongs to the ribokinase family of proteins, has an early evolutionary origin and is highly conserved between species [49], with only a few ADK variants from microorganisms being structurally different [50]. Based on its low $\mathrm{K}_{\mathrm{M}}$ for adenosine $(0.15 \mu \mathrm{M})$ and a highly active substrate cycle between adenosine and AMP, ADK is considered the key enzyme for the regulation of ambient adenosine and minor changes in ADK activity can rapidly translate into major changes in ambient adenosine [51,52]. Therefore, a genetic knockout or knockdown of ADK in cultured cells 


\section{Adenosine metabolism}

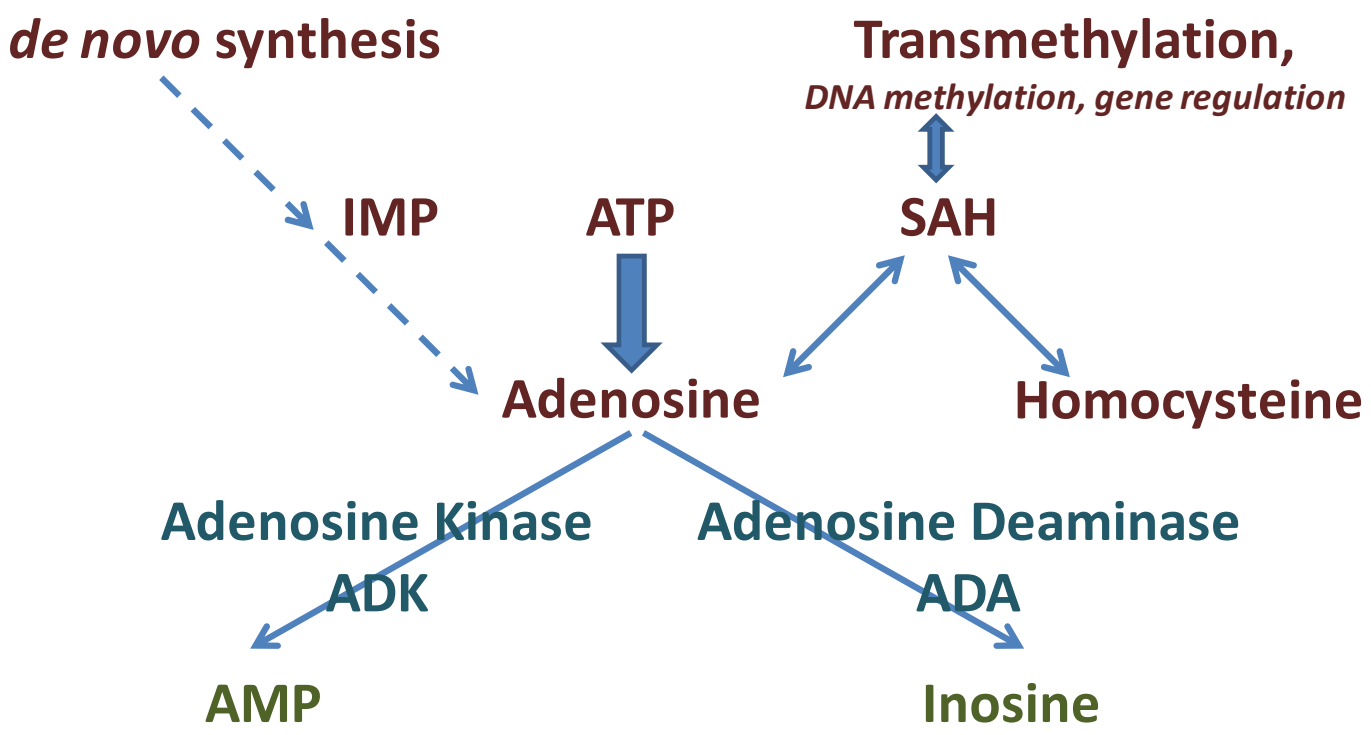

Fig. (1). Major routes of adenosine metabolism.

is a very effective strategy to induce cellular adenosine release [53], a strategy that has therapeutic value [54-56]. ADK appears to be regulated almost exclusively at the protein level. Studies with Leishmania ADK demonstrated that the enzyme is inactivated by aggregation, a conformation that is stabilized by ADP, whereas the active monomer is stabilized by a cyclophilin [57]. In contrast, protein phosphorylation was excluded as a potential mechanism for ADK-regulation [58].

In adult brain, ADK is largely expressed in astrocytes [40], and astrocytic ADK was shown to regulate the basal synaptic levels of adenosine [38]. Under conditions of acute challenge to the brain, e.g. after focal ischemia or during seizures, ADK is rapidly downregulated, likely as a physiological response to raise the protective adenosine-tone [59]. Physiological alterations of ADK have direct impact on brain function. Thus, transgenic overexpression of ADK in brain was shown to be associated with reduced adenosine and seizures [60], with increased susceptibility to stroke [61], with learning impairment and altered susceptibility to psychomimetic drugs [62], and with changes in myelination [63]. Due to its wide implications as a regulator of brain function, the pharmaceutical development of ADK inhibitors has gained much interest as potential anticonvulsant and antinociceptive agents [64, 65].

\section{ADENOSINE KINASE IS OVEREXPRESSED IN EPILEPSY}

As indicated above, astrogliosis is a pathological hallmark of the epileptic brain. Research from our laboratory has shown that astrogliosis in animal models of epilepsy is associated with increased levels of the astrocyte-based enzyme ADK [28, 66, 67]. Likewise, deficient adenosine signalling was found in the hippocampus of kindled rats [68]. The intrahippocampal injection of kainic acid (KA) in mice triggers hippocampal sclerosis and chronic recurrent seizure activity that closely mimics human mesial temporal lobe epilepsy [69-73]. During a time span of 4 weeks affected mice develop profound astrogliosis throughout the injected hippocampus that is associated with overexpression of ADK including an increase of $177 \%$ of enzyme activity compared to control hippocampus resulting in a global reduction of the adenosine-based inhibitory tone [74]. Seizures were suppressed by a low dose of the ADK-inhibitor 5iodotubercidine (ITU) [74]. These findings suggested for the first time that astrogliosis via increasing ADK expression might contribute to the generation of seizures. However, in this complex model of epilepsy it was not possible to segregate the effects of other potential contributors to seizure generation (e.g. mossy fiber sprouting, granule cell dispersion, loss of interneurons) from astrogliosis.

To study selectively the effect of astrogliosis and ADK on seizure generation we generated a mouse model of CA3selective epileptogenesis that develops focal astrogliosis in the absence of other confounding factors such as mossy fiber sprouting or granule cell dispersion [75]. In this model, the unilateral intraamygdaloid injection of KA leads to acute CA3-selective neuronal cell loss that constitutes a trigger for subsequent astrogliosis [75]. Three weeks after KA-injection profound CA3-selective astrogliosis had developed with colocalized overexpression of ADK. Electrographic seizures could be recorded from the astrogliotic CA3, but not from any other brain region [75]. Strikingly, during epileptogenesis seizures coincided temporally with the development of astrogliosis and overexpressed ADK [41]. Seizures in this model could be suppressed by the ADK-inhibitor ITU or by the $\mathrm{A}_{1} \mathrm{R}$ agonist CCPA. Together, these findings suggest that astrogliosis (with overexpression of ADK) is sufficient to trigger seizures in the absence of any other epileptogenetic histopathological alteration. Based on these findings, pharmacological inhibition of ADK should be highly effective in preventing seizures. Indeed, ADK inhibitors are very effec- 
tive anticonvulsant agents that have the potential to amplify endogenous adenosine-signalling thereby minimizing side effects [64, 76-78]. Most importantly, the inhibition of ADK was shown to be effective in a model of pharmacoresistant epilepsy [74].

ADK expression levels have not yet been evaluated in human epileptic brain. However, microdialysis samples indicate lower adenosine baseline levels in epileptic compared to control human hippocampus [79]. Human epileptic brain is also characterized by changes in $\mathrm{A}_{1} \mathrm{R}$ expression and downregulation [80] as well as upregulation [81] of $A_{1} R s$ have been reported.

\section{OVEREXPRESSION OF ADENOSINE KINASE TRIGGERS SEIZURES}

The studies described above show a close association of astrogliosis, overexpression of ADK, and the expression of spontaneous recurrent seizures. To address the question, whether astrogliosis or overexpression of ADK per se are responsible for seizure expression, an ADK transgene was ubiquitously overexpressed in brain (Adk-tg mice) [75]. Transgenic overexpression of ADK was found to be sufficient to trigger spontaneous recurrent electrographic hippocampal seizures at a rate of about 4 seizures per hour, with each seizure lasting about 20 seconds [41]. Most importantly, those seizures were recorded in the absence of any other epileptogenic events such as neuronal cell loss, astrogliosis, mossy fiber sprouting, or granule cell dispersion [41, 75]. To provide a further molecular dissection of astrogliosis from ADK expression levels, Adk-tg mice were subjected to 20 minutes of intraamygdaloid KA-induced status epilepticus (SE). This treatment resulted in a degree of acute seizure severity and corresponding acute neuronal cell loss that was comparable to data from wild-type mice subjected to 30 minutes of SE [82]. The acute KA-induced injury (i.e. trigger for subsequent epileptogenesis) led to an ablation of ADKexpressing CA3 neurons. During a time span of three weeks Adk-tg mice subjected to 20 minutes of SE developed ipsilateral CA3-selective astrogliosis, however without associated overexpression of $\mathrm{ADK}$, whereas the contralateral hippocampus was still characterized by overexpression of transgenic ADK. EEG recordings performed three weeks after KA-injection revealed recurrent electrographic seizures in the ADK-overexpressing contralateral CA3 (in the absence of astrogliosis), but not a single seizure could be detected in the ipsilateral injured CA3 that was characterized by astrogliosis in the absence of overexpressed ADK [82]. These findings further highlight that overexpression of ADK as such rather than astrogliosis is sufficient to trigger seizures. If this assumption is true, then a genetic reduction of ADK should render the brain resistant to seizure development. To test this possibility, fb-Adk-def mice were generated that are characterized by reduced levels of ADK in forebrain amounting to approximately $60 \%$ of total expression levels in matched wild-type mice. These animals were completely resistant to acute KA-induced SE and injury; following intraamygdaloid KA injection, injurious seizures were never observed and not a single TUNEL-positive cell was found 24h after KA-injection [75]. However, when KA was paired with the $\mathrm{A}_{1} \mathrm{R}$ antagonist DPCPX wild-type like seizure activity was restored and the level of the acute CA3-restrictive injury closely matched wild-type controls. Co-injection of
KA with DPCPX permitted us to recreate a wild type-like trigger for subsequent epileptogenesis in fb-Adk-def mice, however, subsequently those animals were under the control of reduced forebrain ADK [75]. Strikingly, three weeks after KA-injection, these animals did not show overt signs of astrogliosis, ADK levels continued to be reduced and no seizures were detected [75]. These findings suggest that a genetic reduction of ADK renders the brain resistant to the development of spontaneous seizures. Therefore, ADK constitutes a valid therapeutic target for the prediction and prevention of seizures in epilepsy. Importantly, ADK regulates basal synaptic adenosine levels and seizure activity, however without affecting activity dependent adenosine-release in hippocampus [38]. Together the studies described above constitute a direct molecular explanation why pharmacological inhibition of ADK is such a powerful tool for seizure suppression [64, 65, 78, 83-85]: because endogenous adenosine levels rise during times of stress [86] (e.g. seizures, lack of oxygen), agents (e.g. the ADK inhibitor ABT-702 [8789]) that amplify this site- and event-specific surge of adenosine could provide antiseizure activity similar to that of adenosine receptor agonists $[64,77]$. Therefore, pharmacological inhibition of ADK is considered to be an efficient tool for the inhibition of epileptic seizures [74, 77] and chronic pain [90]; these successes were associated with an improved therapeutic window compared to $A_{1} R$ agonists [91]. However, systemic application of ADK inhibitors might not be a therapeutic option for epilepsy due to interference with methionine metabolism in liver [92, 93] and the risk of brain hemorrhage [90, 94].

\section{THE ADENOSINE KINASE HYPOTHESIS OF EPILEPSY}

The findings described above form the basis for the ADK hypothesis of seizure generation that has been reviewed previously $[28,66]$. Briefly, any type of injury or stress to the brain (e.g. traumatic brain injury, seizures, or stroke) triggers an acute surge of micromolar levels of adenosine that go far beyond normal levels that are in the 20 to $300 \mathrm{nM}$ range (see review from Bertil Fredholm in this issue). The acute surge in adenosine is likely a combined consequence of increased ATP degradation and decreased adenosine clearance [59, 74]. These high levels of adenosine can constitute a trigger for several downstream effects that all combine to trigger subsequent epileptogenesis. Likely pathways involved are: (i) Changes in adenosine receptor expression levels. Most importantly, a decrease in $\mathrm{A}_{1} \mathrm{R}$ expression and an increase in $A_{2 A} R$ expression on astrocytes influence astrocyte proliferation and may contribute to the development of astrogliosis [95-97]. (ii) Adenosine is an important modulator of the brain immune system [98]. It is well known that inflammatory responses, microglial activation, and changes in the blood brain barrier play early roles during epileptogenesis [22, 99-101]. Thus, an acute surge in adenosine might trigger several immuno-modulatory systems that may contribute to trigger subsequent astrogliosis.

Astrogliosis is a pathological hallmark of several neurological conditions. Studies from our lab have demonstrated that upregulation of $\mathrm{ADK}$ is always a consequence of, but not a cause for, astrogliosis [41, 75, 82]. Astrogliosis, together with upregulation of ADK has not only been observed in animal models of epilepsy, but also in animal models of 
stroke and Alzheimer's disease (Boison, unpublished observations). Therefore it is fair to assume that astrogliotic upregulation of ADK might be a common pathophysiological pathway that might explain for instance the increased incidence of seizures in patients with Alzheimer's disease [102-104] or following stroke [105]. Thus, according to the ADK hypothesis of epileptogenesis, an acute surge of adenosine following any type of brain insult might trigger astrogliosis, which in turn leads to overexpression of ADK and a resulting adenosine deficiency, which - as pointed out above - is sufficient to trigger seizures.

It is important to note that the ADK-associated seizures are frequent, but electrographic and subclinical in nature. ADK-associated seizures in the CA3 model of epileptogenesis can only be recorded with intracranial electrodes and these seizures do not spread, likely because the adenosine system in the vicinity of the epileptogenic focus is still intact $[41,75]$. Interestingly, injection of these animals with a nonconvulsive dose of the $\mathrm{A}_{1} \mathrm{R}$ antagonist DPCPX can turn those electrographic seizures into clinical grade convulsive seizures (Boison, unpublished observations). It is tempting to speculate that ADK-dependent focal electrographic seizures are (i) fairly common, but commonly not diagnosed with surface EEG recording electrodes; and (ii) that these seizures constitute an early event in epileptogenesis requiring secondary events or a secondary "hit", leading to network rewiring or failure of other endogenous antiepileptogenic systems to turn those (undiagnosed?) pre-existing "silent" seizures into clinical seizures. In conclusion, dysfunction of the adenosine system might constitute a very early event in the epileptogenic cascade and targeting the adenosine system is therefore an effective means to prevent seizures in epilepsy, but possibly also to prevent epileptogenesis as such.

\section{FOCAL ADENOSINE AUGMENTATION THERAPIES (AATs) FOR THE TREATMENT OF EPILEPSY}

The paragraphs above suggest that augmentation of the adenosine system is an effective strategy for the prevention of seizures in epilepsy. Based on this neurochemical rationale adenosine augmentation therapies (AATs) have been assessed for therapeutic effectiveness [106-108]. While pharmacological AAT approaches are very effective in preventing seizures (see above), in general those approaches are hampered by significant peripheral and central side effects [106]. To circumvent those side effects focal AATs have been developed with the premise that focal reconstitution of adenosine signalling restricted to a local region of adenosine dysfunction (i.e. the epileptogenic astrogliotic "scar") would restore normal adenosine signalling rather than leading to excessive amounts of adenosine. These strategies are considered to be safe since adenosine is an endogenous anticonvulsant and subject to normal metabolic clearance. Therefore any excessive or detrimental adenosine levels are unlikely to be reached in focal AAT approaches. A proof of principle for this therapeutic concept was first established by implanting adenosine-loaded ethylene vinyl acetate copolymers releasing 20 to $50 \mathrm{ng}$ adenosine per day into the brain ventricles of kindled rats [109]. These polymeric implants provided robust reduction of stage 5 seizures for at least 7 days [109]. To prolong the duration of adenosine release from polymeric brain implants, hamster fibroblasts engineered to release adenosine based on genetic disruption of their $A d k$ gene were encapsulated and thus immuno-isolated into semipermeable polyethersulfone-based hollow fibers and transplanted into the brain ventricles of fully kindled rats. These implants released about $40 \mathrm{ng}$ adenosine per hour and provided almost complete seizure suppression for a duration of 12 days, which corresponded to the life expectancy of the encapsulated cells [110, 111]. The use of encapsulated adenosine releasing myoblasts lead to an extension of the therapeutic time window to up to 8 weeks [112]. In those studies, it was documented that prolonged implant-derived adenosinerelease did not lead to desensitization of adenosine receptors, nor to the development of sedative side effects [112]. The effectiveness of focal adenosine augmentation for seizure suppression was further validated by an independent laboratory that used focal adenosine injections to prevent seizures in rats [113].

In order to provide more refined seizure control, mouse embryonic stem cells were engineered in our laboratory to release therapeutically effective doses of adenosine based on a bi-allelic genetic disruption of the endogenous Adk gene $[114,115]$. These cells were subjected to a defined in vitro differentiation protocol to generate adenosine releasing EScell derived neural progenitor cells that can be transplanted directly into brain without the risk of tumor formation. Direct implants of these cells into the infrahippocampal fissure of immunosuppressed rats formed dense implants within the infrahippocampal fissure and some of the cells migrated into the ipsilateral CA1 region and differentiated into $\mathrm{NeuN}$ positive neurons [56]. Most importantly, these implants effectively suppressed kindling epileptogenesis. The same type of graft prevented the development of epilepsy in the mouse model of CA3-selective epileptogenesis [75].

Embryonic stem cells are ethically controversial and cannot be used as autologous grafts in patients. In order to develop a cell-based system for the focal delivery of adenosine that would be compatible with future clinical applications, human mesenchymal stem cells were engineered to release adenosine using a lentivirus expressing a micro RNA directed against $A d k$. This strategy led to efficient knockdown of ADK and triggered the release of adenosine from these cells [54]. Subsequently, it was shown that infrahippocampal implants of these cells in mice attenuated both acute seizures and acute injury [54], and ameliorated the consequences of KA-induced epileptogenesis [116]. The natural biopolymer silk constitutes an excellent substrate to promote the release of adenosine from stem cells [53]. Thus, silk-based polymers seeded with mesenchymal stem cells derived from a patient and engineered to release adenosine might comprise a viable system for human epilepsy therapy.

To assess the therapeutic potential of silk-based adenosine release, silk based brain implants were engineered to release defined doses of adenosine with a stable release kinetic over several days [117]. Infrahippocampal implants of these constructs provided dose-dependent suppression of kindling epileptogenesis in rats [117]. Silk-based adenosine delivery at a dose of $1000 \mathrm{ng}$ per day was shown to prevent fully kindled seizures, but most importantly novel and powerful antiepileptogenic effects of focal adenosine delivery were demonstrated [118]. 


\section{CONCLUSIONS AND OUTLOOK}

Compelling evidence suggests that a focal dysfunction of adenosine signalling - caused by astrogliosis-induced overexpression of ADK - is sufficient to trigger focal electrographic seizures, possibly a very early event in the epileptogenic cascade that finally leads to the expression of spontaneous recurrent, clinical seizures. Due to the central role of this pathway, focal AAT is highly effective in preventing seizures and likely a viable strategy to prevent epilepsy from developing. Thus, the modulation of adenosinergic signalling is a powerful strategy to interfere with epilepsy on different levels of its genesis. Is this approach ripe for translation into clinical practice?

Human mesial temporal lobe epilepsy is an ideal medical condition for the safe and step-wise introduction of AATs into clinical practice. Patients foreseen for surgical removal of the epileptogenic hippocampus are normally subjected to invasive diagnostics prior to their resection surgery. It would be highly feasible to combine pre-surgical diagnostic evaluation that includes intracranial EEG-recording electrodes with focal intrahippocampal infusion of adenosine. This procedure is feasible since adenosine is already approved by the US Food and Drug Administration (FDA) for intravenous infusion to prevent supraventricular tachycardia. Furthermore, experience is available from phase I clinical trials in which adenosine was infused into the intrathecal space in an attempt to provide a novel strategy for pain control [119121]. These examples demonstrate the safety of adenosine delivery to a human patient. Furthermore, adenosine is an endogenous metabolite and subjected to rapid clearance. Therefore it is highly unlikely that toxic levels of adenosine can be reached. Transient intrafocal adenosine infusion into a patient would allow the following assessments: $(i)$ demonstration that adenosine is effective in preventing epileptiform discharges in a patient with pharmacoresistant epilepsy; (ii) dose escalation studies can be used to identify the most effective dose range; (iii) finally, the epileptogenic hippocampus would be resected and subjected to detailed histopathological analysis. This procedure is considered to be ethically acceptable, since a patient would not be deprived of current standard of care. In a next step, silk-based adenosine releasing polymers could transiently be implanted into the epileptogenic hippocampus (e.g. for several days) prior to its surgical resection. Thus step-wise procedures are possible to implement focal AAT into clinical practice.

Despite this clinical promise there are also critical questions that need to be addressed. Preliminary assessment indicates that focal AATs are not associated with overt side effects [112]. To date however, potential central side effects of focal AATs have not been evaluated systematically, since a stable dose of adenosine over several days or weeks would be necessary for those studies. Future studies are needed to address in detail potential CNS-side effects of focal AAT including psychomotor and cognitive function. Of note is the observation that overexpression of ADK and adenosine deficiency triggers severe cognitive impairment in mice [62]; therefore AATs might eventually be beneficial in ameliorating cognitive impairment, which is a characteristic comorbidity of epilepsy. In addition to the therapeutic potential for epilepsy, AATs might also be useful for the amelioration of adenosine-dependent symptoms in conditions as diverse as chronic pain, Alzheimer's disease, and schizophrenia [27, 122].

\section{ACKNOWLEDGMENT}

The work of the author is supported by grants R01NS058780, R01NS061844, R01MH083973, R21NS057475-01, and R21NS057538-01 from the National Institutes of Health (NIH).

\section{REFERENCES}

[1] White HS, Smith MD, Wilcox KS. Mechanisms of action of antiepileptic drugs. Int Rev Neurobiol 2007; 81: 85-110.

[2] Vajda FJE. Pharmacotherapy of epilepsy: New armamentarium, new issues. J Clin Neurosci 2007; 14: 813-23.

[3] Rogawski MA, Bazil CW. New molecular targets for Antiepileptic drugs: alpha 2 delta, SV2A, and $\mathrm{K}(\mathrm{v}) 7 / \mathrm{KCNQ} / \mathrm{M}$ potassium channels. Curr Neurol Neurosci Rep 2008; 8: 345-52.

[4] Taylor CP, Angelotti T, Fauman E. Pharmacology and mechanism of action of pregabalin: the calcium channel alpha2-delta (alpha2delta) subunit as a target for antiepileptic drug discovery. Epilepsy Res 2007; 73: 137-50.

[5] Brill J, Klocke R, Paul D, et al. Entla: A novel epileptic and ataxic Cacna2d2 mutant of the mouse. J Biol Chem 2004; 279: 7322-30.

[6] Donato R, Page KM, Koch D, et al. The ducky(2J) mutation in Cacna2d 2 results in reduced spontaneous Purkinje cell activity and altered gene expression. J Neurosci 2006; 26: 12576-86.

[7] Vonck K, De Herdt V, Boon P. Vagal nerve stimulation--a 15-year survey of an established treatment modality in epilepsy surgery. Adv Tech Stand Neurosurg 2009; 34: 111-46.

[8] Boon P, Raedt R, de Herdt V, Wyckhuys T, Vonck K. Electrical stimulation for the treatment of epilepsy. Neurotherapeutics 2009; 6: 218-27.

[9] Sharma S, Gulati S, Kalra V, Agarwala A, Kabra M. Seizure control and biochemical profile on the ketogenic diet in young children with refractory epilepsy--Indian experience. Seizure 2009; 18: 4469.

[10] Kossoff EH, Zupec-Kania BA, Rho JM. Ketogenic Diets: An Update for Child Neurologists. J Child Neurol 2009.

[11] Kossoff EH, Rho JM. Ketogenic diets: evidence for short- and long-term efficacy. Neurotherapeutics 2009; 6: 406-14.

[12] Masino SA, Geiger JD. Are purines mediators of the anticonvulsant/neuroprotective effects of ketogenic diets? Trends Neurosci 2008; 31: 273-8.

[13] Bekar L, Libionka W, Tian GF, et al. Adenosine is crucial for deep brain stimulation-mediated attenuation of tremor. Nat Med 2008; 14: 75-80.

[14] Binder DK, Steinhauser C. Functional changes in astroglial cells in epilepsy. Glia 2006; 54: 358-68.

[15] Halassa MM, Fellin T, Haydon PG. The tripartite synapse: roles for gliotransmission in health and disease. Trends Mol Med 2007; 13: 54-63.

[16] Kumaria A, Tolias C, Burnstock G. ATP signalling in epilepsy. Purinergic Signal 2008; 4: 339-46.

[17] Tian GF, Azmi H, Takano T, et al. An astrocytic basis of epilepsy. Nat Med 2005; 11: 973-81.

[18] Kang N, Xu J, Xu Q, Nedergaard M, Kang J. Astrocytic glutamate release-induced transient depolarization and epileptiform discharges in hippocampal CA1 pyramidal neurons. J Neurophysiol 2005; 94: 4121-30.

[19] Lee TS, Mane S, Eid T, et al. Gene expression in temporal lobe epilepsy is consistent with increased release of glutamate by astrocytes. Mol Med 2007; 13: 1-13.

[20] Eid T, Williamson A, Lee TS, Petroff OA, de Lanerolle NC. Glutamate and astrocytes--key players in human mesial temporal lobe epilepsy? Epilepsia 2008; 49 (Suppl 2): 42-52.

[21] Fellin T, Gomez-Gonzalo M, Gobbo S, Carmignoto G, Haydon PG. Astrocytic glutamate is not necessary for the generation of epileptiform neuronal activity in hippocampal slices. J Neurosci 2006; 26 : 9312-22.

[22] Ravizza T, Gagliardi B, Noe F, Boer K, Aronica E, Vezzani A. Innate and adaptive immunity during epileptogenesis and spontaneous seizures: evidence from experimental models and human temporal lobe epilepsy. Neurobiol Dis 2008; 29: 142-60. 
[23] Oberheim NA, Tian GF, Han X, et al. Loss of astrocytic domain organization in the epileptic brain. J Neurosci 2008; 28: 3264-76.

[24] Rouach N, Koulakoff A, Abudara V, Willecke K, Giaume C. Astroglial metabolic networks sustain hippocampal synaptic transmission. Science 2008; 322: 1551-5.

[25] Kang J, Kang N, Lovatt D, et al. Connexin 43 hemichannels are permeable to ATP. J Neurosci 2008; 28: 4702-11.

[26] Szente M. Possible exploitation of non-neuronal and non-chemical synaptic signalling pathways in epilepsy therapy. Curr Signal Transduct Ther 2008; 3: 215-30.

[27] Boison D. Adenosine as a neuromodulator in neurological diseases. Curr Opin Pharmacol 2008; 8: 2-7.

[28] Boison D. The adenosine kinase hypothesis of epileptogenesis. Prog Neurobiol 2008; 84: 249-62.

[29] Pascual O, Casper KB, Kubera C, et al. Astrocytic purinergic signaling coordinates synaptic networks. Science 2005; 310: 113-6.

[30] Abbracchio MP, Burnstock G, Verkhratsky A, Zimmermann H. Purinergic signalling in the nervous system: an overview. Trends Neurosci 2009; 32: 19-29.

[31] Zimmermann H. Extracellular metabolism of ATP and other nucleotides. Naunyn Schmiedebergs Arch Pharmacol 2000; 362: 299309.

[32] Newman EA. Glial cell inhibition of neurons by release of ATP. J Neurosci 2003; 23: 1659-66.

[33] Zhang Z, Chen G, Zhou W, et al. Regulated ATP release from astrocytes through lysosome exocytosis. Nat Cell Biol 2007; 9: 945-53.

[34] Fellin T, Pascual O, Haydon PG. Astrocytes coordinate synaptic networks: balanced excitation and inhibition. Physiology (Bethesda) 2006; 21:208-15.

[35] Halassa MM, Florian C, Fellin T, et al. Astrocytic modulation of sleep homeostasis and cognitive consequences of sleep loss. Neuron 2009; 61: 213-9.

[36] Wall MJ, Atterbury A, Dale N. Control of basal extracellular adenosine concentration in rat cerebellum. J Physiol Lond 2007; 582: $137-51$.

[37] Frenguelli BG, Wigmore G, Llaudet E, Dale N. Temporal and mechanistic dissociation of ATP and adenosine release during ischaemia in the mammalian hippocampus. J Neurochem 2007; 101: 1400-13.

[38] Etherington LA, Patterson GE, Meechan L, et al. Astrocytic adenosine kinase regulates basal synaptic adenosine levels and seizure activity but not activity-dependent adenosine release in the hippocampus. Neuropharmacology 2009; 56: 429-37.

[39] Alanko L, Porkka-Heiskanen T, Soinila S. Localization of equilibrative nucleoside transporters in the rat brain. J Chem Neuroanat 2006; 31: 162-268.

[40] Studer FE, Fedele DE, Marowsky A, et al. Shift of adenosine kinase expression from neurons to astrocytes during postnatal development suggests dual functionality of the enzyme. Neuroscience 2006; 142: 125-37.

[41] Li T, Lan JQ, Fredholm BB, Simon RP, Boison D. Adenosine dysfunction in astrogliosis: cause for seizure generation? Neuron Glia Biol 2007; 3: 353-66.

[42] Zamzow CR, Xiong W, Parkinson FE. Adenosine produced by neurons is metabolized to hypoxanthine by astrocytes. J Neurosci Res 2008; 86: 3447-55.

[43] Geiger JD, Nagy JI. Distribution of adenosine deaminase activity in rat brain and spinal cord. J Neurosci 1986; 6: 2707-14.

[44] Geiger JD, Nagy JI. Ontogenesis of adenosine deaminase activity in rat brain. J Neurochem 1987; 48: 147-53.

[45] Yamamoto T, Geiger JD, Daddona PE, Nagy JI. Subcellular, regional and immunohistochemical localization of adenosine deaminase in various species. Brain Res Bull 1987; 19: 473-84.

[46] Kornberg A, Pricer WE. Enzymatic phosphorylation of adenosine and 2,6-diaminopurine riboside. J Biol Chem 1951; 193: 481-95.

[47] Pak MA, Haas HL, Decking UKM, Schrader J. Inhibition of adenosine kinase increases endogenous adenosine and depresses neuronal activity in hippocampal slices. Neuropharmacol 1994; 33: 1049-53.

[48] Chan T-S, Ishii K, Long C, Green H. Purine excretion by mammalian cells deficient in adenosine kinase. J Cell Physiol 1973; 81: 315-22.

[49] Park J, Gupta RS. Adenosine kinase and ribokinase - the RK family of proteins. Cell Mol Life Sci 2008; 65: 2875-96.

[50] Long MC, Shaddix SC, Moukha-Chafiq O, Maddry JA, Nagy L, Parker WB. Structure-activity relationship for adenosine kinase from Mycobacterium tuberculosis II. Modifications to the ribofuranosyl moiety. Biochem Pharmacol 2008; 75: 1588-600.

[51] Bontemps F, Van den Berghe G, Hers HG. Evidence for a substrate cycle between AMP and adenosine in isolated hepatocytes. Proc Natl Acad Sci USA 1983; 80: 2829-33.

[52] Arch JR, Newsholme EA. Activities and some properties of 5'nucleotidase, adenosine kinase and adenosine deaminase in tissues from vertebrates and invertebrates in relation to the control of the concentration and the physiological role of adenosine. Biochem $\mathrm{J}$ 1978; 174: 965-77.

[53] Uebersax L, Fedele DE, Schumacher C, et al. The support of adenosine release from adenosine kinase deficient ES cells by silk substrates. Biomaterials 2006; 27: 4599-607.

[54] Ren G, Li T, Lan JQ, Wilz A, Simon RP, Boison D. Lentiviral RNAi-induced downregulation of adenosine kinase in human mesenchymal stem cell grafts: a novel perspective for seizure control. Exp Neurol 2007; 208: 26-37.

[55] Pignataro G, Studer FE, Wilz A, Simon RP, Boison D. Neuroprotection in ischemic mouse brain induced by stem cell-derived brain implants. J Cereb Blood Flow Metab 2007; 27: 919-27.

[56] Li T, Steinbeck JA, Lusardi T, et al. Suppression of kindling epileptogenesis by adenosine releasing stem cell-derived brain implants. Brain 2007; 130: 1276-88.

[57] Sen B, Chakraborty A, Datta R, Bhattacharyya D, Datta AK. Reversal of ADP-mediated aggregation of adenosine kinase by cyclophilin leads to its reactivation. Biochemistry 2006; 45: 263-71.

[58] Sahin B, Kansy JW, Nairn AC, et al. Molecular characterization of recombinant mouse adenosine kinase and evaluation as a target for protein phosphorylation. Eur J Biochem 2004; 271: 3547-55.

[59] Pignataro G, Maysami S, Studer FE, Wilz A, Simon RP, Boison D. Downregulation of hippocampal adenosine kinase after focal ischemia as potential endogenous neuroprotective mechanism. J Cereb Blood Flow Metab 2008; 28: 17-23.

[60] Fedele DE, Gouder N, Güttinger M, et al. Astrogliosis in epilepsy leads to overexpression of adenosine kinase resulting in seizure aggravation. Brain 2005; 128: 2383-95.

[61] Pignataro G, Simon RP, Boison D. Transgenic overexpression of adenosine kinase aggravates cell death in ischemia. J Cereb Blood Flow Metab 2007; 27: 1-5.

[62] Yee BK, Singer P, Chen JF, Feldon J, Boison D. Transgenic overexpression of adenosine kinase in brain leads to multiple learning impairments and altered sensitivity to psychomimetic drugs. Eur J Neurosci 2007; 26: 3237-52.

[63] Wu NL, Boison, D. Adenosine kinase expression modulates expression of myelin proteolipid protein. Open Neurosci J 2007; 1: $15-9$.

[64] McGaraughty S, Cowart M, Jarvis MF, Berman RF. Anticonvulsant and antinociceptive actions of novel adenosine kinase inhibitors. Curr Top Med Chem 2005; 5: 43-58.

[65] Boyer SH, Ugarkar BG, Solbach J, et al. Adenosine kinase inhibitors. 5. Synthesis, enzyme inhibition, and analgesic activity of diaryl-erythro-furanosyltubercidin analogues. J Med Chem 2005; 48 : 6430-41.

[66] Boison D. Astrogliosis and adenosine kinase: a glial basis of epilepsy. Future Neurol 2008; 3: 221-4.

[67] Boison D. Adenosine kinase, epilepsy and stroke: mechanisms and therapies. Trends Pharmacol Sci 2006; 27: 652-8.

[68] Rebola N, Coelho JE, Costenla AR, et al. Decrease of adenosine $\mathrm{A}_{1}$ receptor density and of adenosine neuromodulation in the hippocampus of kindled rats. Eur J Neurosci 2003; 18: 820-8.

[69] Riban V, Bouilleret V, Pham-Le BT, Fritschy JM, Marescaux C, Depaulis A. Evolution of hippocampal epileptic activity during the development of hippocampal sclerosis in a mouse model of temporal lobe epilepsy. Neuroscience 2002; 112: 101-11.

[70] Bouilleret V, Schwaller B, Schurmans S, Celio MR, Fritschy JM. Neurodegenerative and morphogenic changes in a mouse model of temporal lobe epilepsy do not depend on the expression of the calcium-binding proteins parvalbumin, calbindin, or calretinin. Neuroscience 2000; 97: 47-58.

[71] Bouilleret V, Loup F, Kiener T, Marescaux C, Fritschy JM. Early loss of interneurons and delayed subunit-specific changes in GABA(A)-receptor expression in a mouse model of mesial temporal lobe epilepsy. Hippocampus 2000; 10: 305-24.

[72] Bouilleret V, Boyet S, Marescaux C, Nehlig A. Mapping of the progressive metabolic changes occurring during the development 
of hippocampal sclerosis in a model of mesial temporal lobe epilepsy. Brain Res 2000; 852: 255-62.

[73] Bouilleret V, Ridoux V, Depaulis A, Marescaux C, Nehlig A, Le Gal La Salle G. Recurrent seizures and hippocampal sclerosis following intrahippocampal kainate injection in adult mice: electroencephalography, histopathology and synaptic reorganization similar to mesial temporal lobe epilepsy. Neuroscience 1999; 89: 717-29.

[74] Gouder N, Scheurer L, Fritschy J-M, Boison D. Overexpression of adenosine kinase in epileptic hippocampus contributes to epileptogenesis. J Neurosci 2004; 24: 692-701.

[75] Li T, Ren G, Lusardi T, et al. Adenosine kinase is a target for the prediction and prevention of epileptogenesis in mice. J Clin Invest 2008; 118: 571-82.

[76] McGaraughty S, Cowart M, Jarvis MF. Recent developments in the discovery of novel adenosine kinase inhibitors: mechanism of action and therapeutic potential. CNS Drug Rev 2001; 7: 415-32.

[77] Kowaluk EA, Jarvis MF. Therapeutic potential of adenosine kinase inhibitors. Exp Opin Investig Drugs 2000; 9: 551-64.

[78] Kowaluk EA, Bhagwat SS, Jarvis MF. Adenosine kinase inhibitors. Curr Pharm Des 1998; 4: 403-16.

[79] During MJ, Spencer DD. Adenosine: a potential mediator of seizure arrest and postictal refractoriness. Ann Neurol 1992; 32: 61824.

[80] Glass M, Faull RL, Bullock JY, et al. Loss of A1 adenosine receptors in human temporal lobe epilepsy. Brain Res 1996; 710: 56-68.

[81] Angelatou F, Pagonopoulou O, Maraziotis T, et al. Upregulation of A1 adenosine receptors in human temporal lobe epilepsy: a quantitative autoradiographic study. Neurosci Lett 1993; 163: 11-4.

[82] Li T, Lan JQ, Boison D. Uncoupling of astrogliosis from epileptogenesis in adenosine kinase (ADK) transgenic mice. Neuron Glia Biol 2008; 4(2): 91-9.

[83] Ugarkar BG, DaRe JM, Kopcho JJ, et al. Adenosine kinase inhibitors. 1. Synthesis, enzyme inhibition, and antiseizure activity of 5iodotubercidin analogues. J Med Chem 2000; 43: 2883-93.

[84] Ugarkar BG, Castellino AJ, DaRe JM, et al. Adenosine kinase inhibitors. 2. Synthesis, enzyme inhibition, and antiseizure activity of diaryltubercidin analogues. J Med Chem 2000; 43: 2894-905.

[85] Wiesner JB, Ugarkar BG, Castellino AJ, et al. Adenosine kinase inhibitors as a novel approach to anticonvulsant therapy. J Pharmacol Exp Ther 1999; 289: 1669-77.

[86] Schrader J, Wahl M, Kuschinsky W, Kreutzberg GW. Increase of adenosine content in cerebral cortex of the cat during bicucullineinduced seizure. Pflugers Arch 1980; 387: 245-51.

[87] Kowaluk EA, Mikusa J, Wismer CT, et al. ABT-702 (4-amino-5(3-bromophenyl)-7-(6-morpholino-pyridin-3-yl)pyrido[2,3-d]pyrimidine), a novel orally effective adenosine kinase inhibitor with analgesic and anti-inflammatory properties. II. In vivo characterization in the rat. J Pharmacol Exp Ther 2000; 295: 1165-74.

[88] Jarvis MF, Yu H, Kohlhaas K, et al. ABT-702 (4-amino-5-(3bromophenyl)-7-(6-morpholinopyridin-3-yl)pyrido[2, 3-d]pyrimidine), a novel orally effective adenosine kinase inhibitor with analgesic and anti-inflammatory properties: I. In vitro characterization and acute antinociceptive effects in the mouse. J Pharmacol Exp Ther 2000; 295: 1156-64.

[89] Suzuki R, Stanfa LC, Kowaluk EA, Williams M, Jarvis MF, Dickenson AH. The effect of ABT-702, a novel adenosine kinase inhibitor, on the responses of spinal neurones following carrageenan inflammation and peripheral nerve injury. Br J Pharmacol 2001; 132: 1615-23.

[90] McGaraughty S, Jarvis MF. Purinergic control of neuropathic pain. Drug Devel Res 2006; 67: 376-88.

[91] Jarvis MF, Mikusa J, Chu KL, et al. Comparison of the ability of adenosine kinase inhibitors and adenosine receptor agonists to attenuate thermal hyperalgesia and reduce motor performance in rats. Pharmacol Biochem Behav 2002; 73: 573-81.

[92] Boison D, Scheurer L, Zumsteg V, et al. Neonatal hepatic steatosis by disruption of the adenosine kinase gene. Proc Natl Acad Sci USA 2002; 99: 6985-90.

[93] Mato JM, Martinez-Chantar ML, Lu SC. Methionine metabolism and liver disease. Ann Rev Nutrition 2008; 28: 273-93.

[94] Erion MD, Wiesner JB, Rosengren S, Ugarkar BG, Boyer SH, Tsuchiya M. Therapeutic potential of adenosine kinase inhibitors as analgesic agents. Drug Dev Res 2000; 50: S14-06.

[95] Hindley S, Herman MA, Rathbone MP. Stimulation of reactive astrogliosis in vivo by extracellular adenosine diphosphate or an adenosine A2 receptor agonist. J Neurosci Res 1994; 38: 399-406.
[96] Brambilla R, Cottini L, Fumagalli M, Ceruti S, Abbracchio MP. Blockade of A2A adenosine receptors prevents basic fibroblast growth factor-induced reactive astrogliosis in rat striatal primary astrocytes. Glia 2003; 43: 190-4.

[97] Rathbone MP, Middlemiss PJ, DeLuca B, Jovetich M. Extracellular guanosine increases astrocyte cAMP: inhibition by adenosine A2 antagonists. Neuroreport 1991; 2: 661-4.

[98] Hasko G, Pacher P, Vizi ES, Illes P. Adenosine receptor signaling in the brain immune system. Trends Pharmacol Sci 2005; 26: 5116.

[99] Vezzani A, Ravizza T, Balosso S, Aronica E. Glia as a source of cytokines: implications for neuronal excitability and survival. Epilepsia 2008; 49(Suppl 2): 24-32.

[100] Uva L, Librizzi L, Marchi N, et al. Acute induction of epileptiform discharges by pilocarpine in the in vitro isolated guinea-pig brain requires enhancement of blood-brain barrier permeability. Neuroscience 2008; 151: 303-12.

[101] Vezzani A, Baram TZ. New roles for interleukin-1 Beta in the mechanisms of epilepsy. Epilepsy Curr 2007; 7: 45-50.

[102] Palop JJ, Mucke L. Epilepsy and cognitive impairments in Alzheimer disease. Arch Neurol 2009; 66: 435-40.

[103] Minkeviciene R, Rheims S, Dobszay MB, et al. Amyloid betainduced neuronal hyperexcitability triggers progressive epilepsy. J Neurosci 2009; 29: 3453-62

[104] Zarow C, Sitzer TE, Chui HC. Understanding hippocampal sclerosis in the elderly: epidemiology, characterization, and diagnostic issues. Curr Neurol Neurosci Rep 2008; 8: 363-70.

[105] Pitkanen A, Kharatishvili I, Karhunen H, et al. Epileptogenesis in experimental models. Epilepsia 2007; 48 Suppl 2: 13-20.

[106] Boison D, Stewart, K-A. Therapeutic epilepsy research: from pharmacological rationale to focal adenosine augmentation. Biochem Pharmacol 2009; doi:10.1016/j.bcp.2009.08.005

[107] Boison D. Adenosine augmentation therapies (AATs) for epilepsy: prospect of cell and gene therapies. Epilepsy Res 2009; 85: 131-41.

[108] Boison D. Engineered adenosine-releasing cells for epilepsy therapy: human mesenchymal stem cells and human embryonic stem cells. Neurotherapeutics 2009; 6: 278-83.

[109] Boison D, Scheurer L, Tseng JL, Aebischer P, Mohler H. Seizure suppression in kindled rats by intraventricular grafting of an adenosine releasing synthetic polymer. Exp Neurol 1999; 160: 164-74.

[110] Huber A, Padrun V, Deglon N, Aebischer P, Mohler H, Boison D. Grafts of adenosine-releasing cells suppress seizures in kindling epilepsy. Proc Natl Acad Sci USA 2001; 98: 7611-6.

[111] Boison D, Huber A, Padrun V, Deglon N, Aebischer P, Mohler H. Seizure suppression by adenosine-releasing cells is independent of seizure frequency. Epilepsia 2002; 43: 788-96.

[112] Güttinger M, Padrun V, Pralong W, Boison D. Seizure suppression and lack of adenosine $\mathrm{A}_{1}$ receptor desensitization after focal longterm delivery of adenosine by encapsulated myoblasts. Exp Neurol 2005; 193: 53-64.

[113] Anschel DJ, Ortega EL, Kraus AC, Fisher RS. Focally injected adenosine prevents seizures in the rat. Exp Neurol 2004; 190: 5447.

[114] Fedele DE, Koch P, Brüstle O, et al. Engineering embryonic stem cell derived glia for adenosine delivery. Neurosci Lett 2004; 370: 160-5.

[115] Güttinger M, Fedele DE, Koch P, et al. Suppression of kindled seizures by paracrine adenosine release from stem cell derived brain implants. Epilepsia 2005; 46: 1-8.

[116] Li T, Ren G, Kaplan DL, Boison D. Human mesenchymal stem cell grafts engineered to release adenosine reduce chronic seizures in a mouse model of CA3-selective epileptogenesis. Epilepsy Res 2009; 84: 238-41.

[117] Wilz A, Pritchard EM, Li T, Lan JQ, Kaplan DL, Boison D. Silk polymer-based adenosine release: Therapeutic potential for epilepsy. Biomaterials 2008; 29: 3609-16.

[118] Szybala C, Pritchard EM, Wilz A, Kaplan DL, Boison D. Antiepileptic effects of silk-polymer based adenosine release in kindled rats. Exp Neurol 2009; 219: 126-35.

[119] Eisenach JC, Curry R, Hood DD. Dose response of intrathecal adenosine in experimental pain and allodynia. Anesthesiology 2002; 97: 938-42.

[120] Eisenach JC, Hood DD, Curry R. Preliminary efficacy assessment of intrathecal injection of an American formulation of adenosine in humans. Anesthesiology 2002; 96: 29-34. 
[121] Eisenach JC, Hood DD, Curry R. Phase I safety assessment of intrathecal injection of an American formulation of adenosine in humans. Anesthesiology 2002; 96: 24-8.
[122] Boison, D. Adenosine as a modulator of brain activity. Drug News Persp 2007; 20: 607-11

Received: December 02, 2009

(C) Detlev Boison; Licensee Bentham Open.

This is an open access article licensed under the terms of the Creative Commons Attribution Non-Commercial License (http://creativecommons.org/licenses/by-nc/3.0/) which permits unrestricted, non-commercial use, distribution and reproduction in any medium, provided the work is properly cited. 